\title{
Carrying forward the Uses and Grats 2.0 agenda: An affordance-driven measure of social media uses and gratifications
}

\author{
(Accepted manuscript- Journal of Broadcasting and Electronic Media) \\ Chamil Rathnayake, University of Strathclyde, UK \\ Jenifer Sunrise Winter, University of Hawaii, USA
}

\begin{abstract}
The notion of social media affordances has not been adequately integrated into the uses and gratifications literature. Building on the MAIN model, an affordance-driven framework (Sundar, 2008; Sundar \& Limperos, 2013), this study develops a social media uses and gratifications scale among a sample of 393 students. Results of the study support the conceptual accuracy of the MAIN model, as conceptualizing social media uses and gratifications as a second-order factor structure that classifies constructs into four different types of affordances displays similar goodness-of-fit to a single-order factor structure. A confirmatory factor analysis with a second sample of 313 American citizens further confirms the applicability of the scale among a more diverse population.
\end{abstract}




\section{Introduction}

Uses and Gratifications (U\&G) is a much-debated theoretical approach that has its origins in media effects studies (Ruggiero, 2000). This approach has direct implications for understanding the dynamics of social media consumption. Examining social media uses and gratifications requires identifying unique uses and gratifications, developing and validating measures, and testing them across different user groups and platforms. Several early studies highlighted the importance of examining uses and gratifications in the context of computermediated communication (e.g., Ruggiero, 2000). There is a growing body of literature examining various aspects of uses and gratifications in the context of social media, such as social network sites (e.g., Alhabash, Chiang, \& Huang, 2014; Apaolaza, He, \& Hartmann, 2014; Chen \& Kim, 2013; Lan-Ying, Hsieh, \& Wu, 2014; Pai \& Arnott, 2013; Smock, Ellison, Lampe, \& Wohn, 2011), online games (e.g., Wu, Wang, \& Tsai, 2010), micro-blogs (e.g., Chen, 2011), crowdsourced business review sites (e.g., Hicks et al., 2012), social recommendation systems (e.g., Kim, 2014), web-based information services (e.g., Luo \& Remus, 2014), and social media in general (e.g., Leung, 2013; Wang, Tchernev, \& Solloway, 2012). Although the body of social media $U \& G$ literature is diverse in terms of measures used, and platforms and populations examined, many studies tend to use more user-centered measures such as socializing, entertainment, information sharing, escapism, need to connect, and convenience (e.g., Apaolaza et al., 2014; Chen, 2011; Hicks et al., 2012; Lan-Ying et al., 2014; Smock et al., 2011). This has resulted in a dearth of studies that consider new affordances of social media. Social media uses and gratifications also depend on the design of the platforms. Moreover, the measures used in recent social media U\&G studies are also varied, leading to inconsistencies. Social media researchers need to acknowledge this issue and develop and test measures that consider both 
users, as well as the affordances, of platforms. Sundar and Limperos (2013) identify this issue and claim that new media studies tend to be biased towards social and psychological factors rather than medium-related factors. They suggest that researchers need to focus on the technologies themselves and the new uses and gratifications they create. They suggest sixteen types of gratifications based on four classes of social media affordances (Modality, Agency, Interactivity, and Navigability) and items that can be used to measure those gratifications. In addition to the conceptualization, they suggest possible measures to operationalize these constructs. Validating this measure, and testing it across different actors and attributes, will help strengthen the body of U\&G literature. Therefore, an important first step in examining the dynamics of social media uses and gratifications is to validate the Uses and Grats 2.0 constructs.

This study develops a measure of social media uses and gratifications using the conceptualizations suggested by Sundar and Limperos (2013). The scope of the study extends beyond a mere validation of the original model and items suggested, building on the original framework by refining the items, introducing new items specifically tailored to social media, and testing them to develop a measure of social media uses and gratifications.

\section{Social Media Uses and Gratifications}

The new media U\&G literature ranges from studies that examine effects of uses and gratifications (e.g., Chen \& Kim, 2013; Apaolaza et al., 2014), work that examines moderating and mediating effects (e.g., Chen, 2011; Huang et al., 2014), and research that operationalizes uses and gratifications measures (e.g., Kaye, 2010). While some scholars examine uses and gratifications in the context of practices such as online gaming (e.g., Wu et al., 2010) and blogging, social network sites have been the center of attention among U\&G scholars. Pai and Arnott (2013) highlight the need to differentiate social network sites from other online 
communities and claim that users adopt them to attain four values (belonging, hedonism, selfesteem, and reciprocity). Ancu and Cozma (2009) claim that social interaction is the primary motive for accessing profiles of political candidates, while entertainment and informationseeking are secondary. Quan-Haase and Young (2010) argue that Facebook users tend to seek enjoyment and knowledge about the social activities within their networks, while instant messaging is focused more on relationship building and maintenance. Smock et al. (2011) suggest that, while only three motivations predict general Facebook use, a relatively higher number of variables can predict motivations for using specific features such as status updates, comments, chat, and groups. Krause, North, and Heritage (2014) examine the motives for listening to music on Facebook and identify three gratifications: entertainment, communication, and habitual diversion. These studies focus on explaining uses and gratifications unique to the Internet and social media.

\section{The Need for Platform-oriented Measures}

The body of social media uses and gratification literature, in general, pays more attention to identifying uses and gratifications, and examining gratifications that play a primary role in social media use. This does not necessarily include attempts to identify gratifications unique to social media. As social media are different from traditional media, they may be able to generate new uses and gratifications. Accordingly, social media U\&G studies need to focus on at least two aspects. First, it is necessary to evaluate the recent social media U\&G literature in terms of its coverage of unique uses and gratifications. Second, these measures need to be evaluated in terms of their coverage of user-based and platform-based gratifications.

The notion of social media affordances has recently gained the attention of social media scholars. Generally defined, affordances relate to the mutuality between social media users and 
features of the platforms that enable or constrain behavior. Majchrzak, Faraj, Kane, and Azad (2013) identify technology affordances as "the mutuality of actor intentions and technology capabilities that provide the potential for a particular action" (p. 39). According to boyd (2011), affordances can destabilize core assumptions related to engaging in social life and reshape publics directly and indirectly. boyd notes that affordances such as persistence, replicability, scalability, and searchability of online content can play a significant role and help scholars understand why people engage the way they do. This concept is important for social media studies, as affordances can structure user actions by enabling or constraining them, thereby affecting social media uses and gratifications.

Sundar and Limperos (2013) discuss new media uses and gratifications and stress the importance of conceptualizing affordance-based uses and gratifications. They highlight that new media $U \& G$ studies have only slightly modified older media gratifications to suit new media, and examine 20 studies published between 1940 and 2011 to reveal overlaps in measures across different types of new media. They argue that nuanced, and perhaps new, gratifications have not been fully specified. The collection of studies they reviewed covers a broad range of technologies or media, such as radio talk shows, telephone, newspapers, TV, video games, and social network sites. While these studies help the authors to argue that affordance-based measures are necessary, they may not represent what is commonly known as social media. Therefore, prior to adopting their framework and items for scale development, we support their argument with a more thorough examination of recent measures used in $U \& G$ studies that focus on social media, in order to ascertain whether these studies include gratifications that can characterize social media. 
Previous U\&G studies have used a considerably diverse set of measures that capture the uniqueness of social media. Social media studies should take into consideration aspects such as the interactive nature of social media platforms, the ability of users to create and manage content, and the wide variety of features available. Measures such as socializing (Apaolaza et al., 2014), virtual community (Chen \& Kim, 2013), socialization-seeking (Kim, 2014), interpersonal utility (Luo \& Remus, 2014), reciprocity (Pai \& Arnott, 2013), expressive information sharing, companionship, professional advancement, social interaction, meeting new people (Smock et al., 2011), career opportunities, global exchange (Roy, 2009), surveillance (Zhang \& Zhang, 2013), self-status seeking (Park, Kee, \& Valenzuela, 2009), and spiritual support, psychological support, and networking (Anderson, 2011) cover a broad range of unique gratifications.

Social media uses and gratifications can be classified as either user-oriented or platformoriented. User-orientation puts less emphasis on the features or affordances of the platform. For instance, socializing, spiritual support, and community building gratifications focus only on the user. Platform-oriented uses and gratifications take into consideration the features of the platform or the affordances they offer. For example, blog ambiance gratification (Kaye, 2010) acknowledges that the users enjoy the overall atmosphere of a blog. Although the measures used in previous studies adequately cover user-oriented uses and gratifications (e.g., socializing, psychological support, and surveillance) that have social and psychological origins, they lack coverage of social media/platform-oriented measures. Therefore, the argument made by Sundar and Limperos (2013) that there is a lack of emphasis on new media affordances is valid in particular for contemporary social media studies. Social media result in platform-oriented gratifications, as there is a range of social media platforms with many features and functionality. Therefore, users may be motivated by the nature of the platform itself. Factors such as design 
may provide gratifications for the users. For instance, navigating a site itself can be psychologically rewarding. The responsiveness of the platform may fulfill the need of the user. This argument highlights the need for affordance-based measures for social media.

The MAIN Model (Sundar, 2008) provides a solid conceptual foundation to examine social media uses and gratifications while considering the effects of affordances. This model suggests that four affordances (Modality, Agency, Interactivity, and Navigability) are prevalent in contemporary digital media, and they are able to cue cognitive heuristics (i.e., judgment rules) that can lead to credibility assessments. These are related to design features and characteristics that affect user impression of the media. Sundar argues that each user is different in terms of the meanings he or she makes from affordances. The model suggests that modality, agency, interactivity, and navigability cues embedded in affordances are significant in shaping user assessment of the medium. This perspective suggests that the sheer presence of these affordances results in users experiencing media content in a certain way. Sundar and Limperos (2013) suggest 16 gratifications that relate to four broad classes of affordances (Table 1).

\section{[TABLE 1]}

In general, the MAIN Model rejects the idea that all gratifications relate to innate needs and argues that new and distinctive gratifications can emerge from new media affordances. The MAIN Model identifies Modality, Agency, Interactivity, and Navigability as four types of affordances that can lead to different gratifications. It emphasizes the capability of a medium to facilitate certain actions and suggests that the user is an integral element as he or she interprets the affordance.

Modality, defined by Sundar and Limperos as "different methods of presentation (e.g., audio or pictures) of media content, appealing to different aspects of the human perceptual 
system (e.g., hearing, seeing)", acknowledges that the Internet can provide users with content in multiple modalities and some of them can be considered unique (e.g., pop-up ads) (p. 512). The Agency affordance of the MAIN Model suggests that the Internet allows users to be agents or sources of information, and this view acknowledges the ability of users to be gatekeepers of content, build communities, and contribute. As Sundar (2008) explains, the Agency affordance acknowledges that in digital media the source can be attributed to a computer, a location (e.g., a website), or even other users. Interactivity, according to Sundar, is the most distinctive affordance of digital media, and it relates to interaction and activity on a given medium. Sundar and Limperos (2013) define Interactivity as "the affordance that allows the user to make realtime changes to the content in the medium" (p. 515), and Navigability as "the affordance that allows user movement through the medium" (p. 516). Sundar (2008) notes that metaphors like "site" and "cyberspace", the availability of navigational aids and content generated, and even the mere presence of navigational aids, such as hyperlinks, can trigger certain heuristics. Sundar and Limperos argue that the MAIN Model captures the uniqueness of new media and suggest a set of potential items to measure each gratification in the model. This model is suitable to examine social media uses and gratifications due to several reasons. First, it focuses on the capacity of the Internet-based platforms to provide a range of usage or engagement options. Second, it suggests platform-oriented gratifications that have not been discussed in previous studies. Finally, the model is comprehensive. Therefore, the MAIN Model provides a rich approach to understand new media uses and gratifications. However, it has not yet been subject to adequate academic inquiry, validated, and tested across different populations.

\section{Method}

Item development 
For this study, social media were defined as online platforms where users can interact with each other, build networks, share and create content, publish, and make comments. Social media include a range of platforms, such as Facebook, Twitter, YouTube, Instagram, Tumblr, Reddit, and WordPress. The survey instrument provided a definition of social media to respondents to ensure that they reflected on interactive platforms without restricting their responses to a specific platform.

The set of 57 items suggested by Sundar and Limperos (2013) was used as the initial pool of items for the scale. As their items are not targeted towards a particular technology, contain general statements, and focus on devices, they were re-written specifically to focus on social media. For instance, the item "I feel like I am able to experience things without actually being there" was re-written as "Social media provide quality information that makes me feel like I am able to experience things as they are without actually being there." In addition, 15 new items were introduced. For instance, items such as "social media help me to have real interactions with people although I am not in physical proximity" and "Social media allow me to actively contribute to communities that make an impact on society" were added to capture the uniqueness of social media. The final set of items included 72 statements tailored to measure the constructs in the context of social media. The items were checked by three independent readers to evaluate the clarity and meaning.

\section{Data Collection}

Two online surveys were conducted to collect data for the study. The first survey was conducted among students in 15 classes at three campuses in the State of Hawaii. Surveys with missing entries were excluded, yielding a sample for analysis consisting of 393 respondents. The demographics of the sample paralleled the demographics of the universities. Students of all grade 
levels were sampled, including graduate students. The sample was appropriate for examining social media uses and gratifications, as more than $90 \%$ of the subjects indicated that they use social media at least once a day. A second survey was conducted through a professional agency to collect data from a more diverse and representative sample. The second sample included 313 American citizens representing different age groups, education levels, and ethnic groups.

\section{Identification and Refinement of Latent Constructs}

Four exploratory factor analyses (maximum likelihood with Varimax rotation) were conducted using the first sample to identify the best items to measure constructs representing each class of affordance. Factor analysis was repeated, after dropping weak items, until an acceptable solution was reached. This analysis resulted in a reduced pool of 42 items that had high correlations (at least .5, except for a few items) with the factors that they were hypothesized to operationalize. Several items with loadings below .50 were retained as they had loadings close to .50 and at least three items were used to operationalize each construct. Several items that loaded on to a different factor and had low cross-loadings were identified as new items that could operationalize the latter factor.

\section{Assessment of the Latent Factor Structure}

The items used for exploratory factor analysis (EFA) were hypothesized to exhibit a latent factor structure that corresponds to Sundar and Limperos's (2013) conceptualization of affordance-based uses and gratifications. The EFA provides a preliminary test to determine the items that best reveal latent factor structure for each MAIN dimension. These results were verified by conducting a confirmatory factor analysis (CFA), ensuring that a factor structure that includes all constructs shows reasonable fit. The theoretical foundation provided by the MAIN model, however, extends beyond a first-order factor structure, as the constructs represent four 
different classes of affordances. Therefore, a second-order factor structure was tested to examine whether the constructs suggested by initial analysis correspond with the MAIN model. CFAs focused on validating the scale along two dimensions: 1) social media uses and gratifications as a first-order factor structure that examined the goodness-of-fit of the operationalization of constructs, and 2) a second-order factor structure that examines the goodness-of-fit of classifying constructs tested in the first model under the four classes of affordances suggested in the MAIN model. This two-step analysis allowed examination of the suitability of individual items to operationalize each construct as well as constructs to represent each class of affordance. An additional CFA was conducted with the second sample to test the validity of the scale further.

\section{Results and Discussion}

\section{Identification of Latent Constructs}

Factor loadings provided in Table 2 show that the factors identified in each solution suggest several distinct dimensions for each class of affordance. While these models support the original conceptualization, they indicate some deviations from the original factors and items suggested by Sundar and Limperos (2013). These deviations, and the factor structure in general, provide insight into understanding user perceptions of social media.

\section{[TABLE 2]}

Results of the factor analyses show factors and items that can conceptualize social media uses and gratifications. The first factor analysis provided a three-factor solution (Realism, Coolness, and Being There) that included two newly introduced items (REAL 4: I can experience the real world through social media, and BEIN 5: Social media help me to have real interactions with people although I am not in physical proximity). BEIN 5 was retained despite low loading, as it can allow more modifications in the CFA. Coolness and Novelty items also 
loaded into a single factor after weak items were excluded. The survey included five and four items to measure Coolness and Novelty respectively. However, the solution given in Table 2 includes two Coolness items and three Novelty items loaded into one factor. Coolness, as Sundar, Tamul, and Wu (2014) found, includes several dimensions, such as attractiveness, originality, and sub-cultural appeal. Therefore, capturing the essence of Coolness is challenging at best. While both Coolness items suggested by Sundar and Limperos (2013) and the newly introduced items capture aspects of Coolness, Novelty items triggered a very similar perception among respondents. This might result from the striking similarity between the two factors. Sundar et al. (2014) note that "an interface is cool if it is novel" (p. 177). For instance, distinctiveness (COOL2) and differences in interface (NOVL3) are highly related so that they load into one factor. Accordingly, combining Coolness and Novelty into one factor does not jeopardize the quality of the model.

Operationalizing uses and gratifications related to agency-enhancement is important as social media are often associated with engagement and participation. This is particularly the case with regards to political action. The survey provided a four-factor solution (Agency, Community Building, Bandwagon, and Filtering), which is slightly different from the original five-factor conceptualization, to measure agency-based social media uses and gratifications. This solution builds on the work of Sundar and Limperos (2013), as the items retained include five items that were newly introduced to the scale (AGNC1: social media allow me to freely express my opinions, CMNB3: social media allow me to build a network that could bring me social support, BAND4: reading others comments on social media before I make comments helps me to avoid potential conflicts, BAND5: I try to adjust my reactions to social media posts based on comments made by others, FILT4: social media allow me to limit the visibility of information I 
post to a small group). These items were specifically written to capture the ability of social media to engage users and facilitate action. Moreover, several items in the original list were significantly changed to fit with social media, including the ability to facilitate formation of social groups beyond geographic boundaries.

A main difference between the original conceptualization and this factor structure was that Filtering and Ownness did not load into distinct factors. Most of the Ownness items had high cross-loadings and were removed to improve the factor solution. However, OWNN 3 (my friends have their own ways of using social media) loaded into Filtering with a reasonable value and very low cross-loadings. This item was retained despite its low loading, as it helps operationalize the construct with at least three items, and it had a higher loading than other Filtering items. This is not a limitation, as it guides further discussion on differences between Filtering and Ownness. As with Coolness and Novelty, it is difficult to distinguish between Filtering and Ownness. Filtering may result in a sense of Ownness, as the facility to sort through information (FILT3) and limit the visibility of information (FILT4) can result in unique uses that make users feel like their friends have their own ways of using social media (OWNN3).

While most of the items in the interactivity-based uses and gratification factor model were the same as, or some variation of, the items suggested by Sundar and Limperos (2013), three new items (ACTV1: I can perform a number of tasks on social media, ACTV4: I get to do a lot of things on social media, and DYNM2: social media give me more control over information I post) had high loadings to support the factor structure. In contrast to the original four-factor conceptualization, this model suggested three latent factors to operationalize interactivity-related uses and gratifications of social media. Activity had clear and reasonably high loadings that make it a distinct factor in the model. However, the model did not support identifying 
Responsiveness and Dynamic Control as two separate factors. This does not undermine the validity of the model, as it is reasonable to use both to operationalize a single factor since Responsiveness is a necessary condition for Dynamic Control. Responsiveness of social media to commands (RESP2) and requests (RESP3) can trigger the perception that users are in charge (DYNM1), have more control over information (DYNM2), and are able to control the interaction (DYNM3).

Uses and gratifications related to Navigability cover Browsing, Scaffolding/Navigation Aids, and Play/Fun aspects. While the first two aspects are related, Play shows a different dimension of navigation. Results (Table 2) reflect those nuances, as Browsing/Variety Seeking and Scaffolding/Navigation Aids converged into one factor. This shows that participants' evaluation of the two is not different. This is not conceptually counter-intuitive, as Scaffolding can be part of Browsing. For instance, the ability of social media to link users to sites that have different types of information (BROW2) and other pieces of information (SCAF2) are very similar. Visual aids available on social media (SCAF3) help users to obtain a variety of information (BROW1). Due to the highly related nature of these two concepts, and with the support of factor loadings given in Table 2, they can be conceptualized as a single factor. Play, being a distinct dimension, loaded into a different factor. Consequently, the solution was a twofactor solution that was somewhat different from the hypothesized three-factor solution. Three out of nine items in the model (BROW2: social media can link me to sites that have different types of information, PLAY4: social media provide more entertaining information than other media, and PLAY5: social media offer more entertaining features than other media) were newly introduced, and these items focus on accessibility of information, entertainment provided by information, as well as features of social media. 
The correlation matrix given in Table 3 shows that, except for four relationships (Realism and Coolness, Realism and Filtering, Realism and Interaction, and Realism and Browsing), latent constructs identified by the separate factor analyses significantly correlate with each other. These correlations, however, are low to moderate, ranging from 0.120 to 0.568 . This shows that the constructs have reasonable convergent validity in terms of representing the broad class of affordance they are hypothesized to measure. Low to moderate correlations between constructs also support discriminant validity, as they are not overly correlated. Significant correlations among constructs across classes of affordances also indicate that the constructs and items can represent a single scale that has multiple dimensions to measure social media uses and gratifications. This supports conducting an all-inclusive factor analysis to support the validity of the model.

\section{[TABLE 3]}

\section{Assessment of the Latent Factor Structure}

The above analysis supports, subject to the revisions discussed above, Sundar and Limperos' (2013) conceptualization of uses and gratifications under each class of affordance. Table 4 shows a revision to their conceptual framework based on the EFA results discussed above. Except for these changes, the revision suggested by the EFAs does not differ from the original conceptualization. Although the results did not distinguish between several factors, no items loaded into factors that represent a different class of affordance. This indicates that, while there is some room to refine the measures within each affordance, the distinction between affordances can be supported in the context of social media. As this was not accomplished in the separate EFAs, further analysis was necessary to test the validity of the whole model. Therefore, 
the revised conceptual framework was used to test several CFA models to evaluate goodness- offit of the latent factor structure.

\section{[TABLE 4]}

The latent factor structure summarized in Table 4 was evaluated in two steps using three CFA models: 1) social media uses and gratifications constructs as a first-order factor structure, 2) as a second-order factor structure that includes the MAIN dimensions, and 3) a model that tests the resultant second-order factor structure with the second sample. The first model situates the results of the four separate EFAs discussed above in a single factor structure that can support the validity of an all-inclusive model. This is important, as it shows the suitability of individual items to operationalize each construct regardless of the affordance they represent. The second model was necessary, as it tests perhaps the most important contribution made by the original model (i.e., that uses and gratifications can represent four main types of affordances). This shows the suitability of each construct to support broad affordances suggested by Sundar and Limperos (2013), supported by the EFAs, as well as provides empirical evidence to distinguish between the four types of affordances. Results of the EFAs were used to build an all-inclusive first-order confirmatory factor model and a second-order factor model. Three criteria were used to further improve the model fit: 1) standardized regression weights, 2) standardized residuals, and 3) modification indices. Several items with high standardized residuals and low standardized regression weights were dropped and covariances were drawn between two adjacent items to further improve the model fit. On an individual parameter level, all CFA models showed acceptable results - standardized regression weights ranged between plus and minus one, standard errors were greater than zero and less than 0.20 for almost all parameters, and all parameters in both models were statistically significant $(\mathrm{p} \leq 0.05)$. 
According to $\mathrm{Hu}$ and Bentler (1999), cut-off values close to .95 for the Tucker Lewis Index (TLI) and Comparative Fit Index (CFI) and .06 for the Root Mean Square Error Approximation (RMSEA) indicate reasonable fit. The first CFA model tested a single-order factor structure and included 33 items and indicated reasonable fit: root mean square residual (RMR): 0.049, Goodness-of-Fit Index (GFI): 0.878, Adjusted Goodness-of-Fit Index (AGFI): 0.844, Incremental Fit Index (IFI): 0.910, TLI: 0.892, CFI: 0.910, RMSEA: 0.052. The model had high standardized regression weights for each item. These results support using the factors identified in the four separate EFAs above as distinct constructs in an all-inclusive model. Given the acceptable fit of the first CFA model, a second CFA was conducted to examine the suitability of the constructs to represent each affordance in the MAIN model. Items used in the first CFA were used to develop this model, and second-order items were introduced to operationalize the MAIN dimensions. Items with high standardized residuals and low standardized regression weights were dropped to further improve the model fit. This model also consisted of 33 items and showed reasonable fit (RMR: 0.056, GFI: 0.854, AGFI:0.828, IFI: 0.888, TLI: 0.875, CFI: 0.887, RMSEA: 0.056). This indicates that Sundar and Limperos's (2013) conceptualization of affordance-based uses and gratifications is valid as a second-order factor structure. It is notable that there are no major differences in regression weights between the two models. However, one weak item in this model (INTR1) negatively affected the overall model fit. This item was retained in the EFA and in this CFA as operationalizing Interaction with at least three items could help future studies that use this scale to further refine the items that represent the construct. For each model, all but two relationships had weights lower than 0.50 . Most of the effects in the models had weights higher than 0.60. In general, standardized regression weights and the modelfit indices show that conceptualizing social media uses and gratifications under the four types of 
affordances suggested by Sundar and Limperos (2013) does not jeopardize the statistical validity of the measure. Coolness and Novelty items were excluded from the model, as Coolness included two Novelty items and users may not perceive many of the social media platforms as novel anymore. This can particularly be the case with student populations that are generally viewed as heavy social media users. The resultant factor structure was further tested with the second sample to ensure that it can be applied for different populations. We excluded Interaction from the final model to maximize the validity of the model. This, however, does not mean that this construct should be excluded in future applications of the scale, as larger samples and refined items can help improve the construct. The revised model (Figure 1) showed slight improvement (RMSEA: 0.054, RMR: 0.048, GFI: 0.852, AGFI: 0.822, IFI: 0.931, TLI: 0.922, CFI: 0.931), indicating that the measure suggested can produce similar results when tested with a different, and more diverse, sample.

\section{[FIGURE 1]}

Table 2 shows means, standard deviations, and Cronbach's alpha values for each construct. The results indicate that, while respondents disagree that social media is similar to real life (mean: 2.03 on a five-item Likert scale), their perception of other uses and gratifications constructs range between three (neither agree nor disagree) and four (agree). However, the fact that the mean values of these responses gravitate towards four (e.g., Coolness: 3.75, Community Building: 3.68, Filtering: 3.83, and Browsing: 3.80) shows that the revised items capture uses and gratifications relevant to social media. Standard deviations are well below one, showing that there are no issues of normality in the sample. Moreover, alpha values above 0.64 indicate that the items have reasonable internal consistency. 
A Multivariate Analysis of Variance (MANOVA) was conducted to provide a perspective on the effects of demographic variables. This test examined the effects of gender, age, ethnicity, and education of respondents on their perception of uses and gratifications. The results showed that, while gender, age, and ethnicity have no significant effect, the perception of social media uses and gratifications can be affected by the education level of respondents (Pillai's Trace: $0.223, \mathrm{~F}: 1.431, \mathrm{p} \leq 0.05)$. The between-subjects effects showed that perception of Agency and Filtering can depend on education level (Agency: F: 3.35, $\mathrm{p} \leq 0.05$, and Filtering: F: 4.328, $\mathrm{p} \leq 0.05)$. This is possible, given the presence of graduate students in the sample.

The two interaction effects tested in the model (Age $\times$ Education and Age $\times$ Ethnicity) were significant, indicating that the impact of age on perception of social media uses and gratifications depends on the education level (Pillai's Trace: 0.603, F: 1.659, $\mathrm{p} \leq 0.05$ ) and ethnicity of respondents (Pillai's Trace: 0.386, F: 1.248, p $\leq 0.05$ ). According to between-subjects effects, the impact of age on several uses and gratifications constructs (e.g., Being There: F: 2.30, $\mathrm{p} \leq 0.05$, Agency: $\mathrm{F}: 3.43, \mathrm{p} \leq 0.05$, Filtering: $\mathrm{F}: 2.55, \mathrm{p} \leq 0.05$, Responsiveness: $\mathrm{F}: 2.13, \mathrm{p} \leq$ 0.05, Play: F: $1.83, p \leq 0.05)$ depends on the education level of respondents. Similarly, the results showed that the impact of age on two dependent variables (Realism: F: 2.07, p $\leq 0.05$, Agency: F: 2.54: $\mathrm{p} \leq 0.05$ ) depend on the ethnicity of participants.

The fact that basic demographic variables, such as gender, age, and ethnicity, have no direct effect on uses and gratifications indicates that the perception of constructs is consistent within the sample. However, the results show that there can be nuances, rather than direct differences, in the perception of social media uses and gratifications. For instance, significant interaction effects show that the impact of age on perception of uses and gratifications depends on the educational level and ethnicity of respondents for some constructs. This leaves room for 
further examination of social media uses and gratifications in different settings with special attention on effects of different variables on specific constructs.

\section{Conclusions}

Testing an affordance-driven measure of social media uses and gratifications using the conceptual framework and items suggested by Sundar and Limperos (2013) requires achieving two main goals: 1) testing the appropriateness of items (or revisions) to operationalize constructs, and 2) examining the statistical validity of classifying constructs tested in the first step into four types of affordances conceptualized by the original study. This requires moving beyond a more conventional single-order scale development to testing a scale that has two levels (constructs and a broad classification of these items). This study, using two different samples, achieves these two goals. Sundar and Limperos's framework is conceptually rich, and it has practical suggestions for operationalization. However, the measure they suggested needs refinement for application in the context of social media. The results of this study support the conceptual accuracy of the MAIN model, as they indicate that conceptualizing social media uses and gratifications as a second-order factor structure that classifies constructs into four different types of affordances does not jeopardize the statistical validity of the measure. The distinction between the two factor structures is mainly theoretical as the objective this study is to add the notion of social media affordances to uses and gratifications studies. This, however, does not mean that the scale suggested should not be used as first-order factor structure. Such use still provides a more comprehensive measure of uses and gratifications as it has been developed based on social media affordances. Using this scale as a second-order structure allows more advanced analysis that includes affordances as second-order factors. 
Rather than treating the results of the present study as a mere validation of the items suggested by Sundar and Limperos (2013), the factor structure and the measures tested can be considered an improved scale to measure social media uses and gratifications. This is appropriate for several reasons: 1) the original items suggested by Sundar and Limperos (2013) were not targeted towards social media; 2) the original items were subject to substantive revisions in this study to tailor them to social media users; 3) a reasonable number of new items were added, and ten of them were included in the final thirty-item scale; and 4) several constructs suggested by the original study were combined to create new constructs, making the present framework a reduced version of the original conceptualization. However, the essence of the MAIN framework remains intact in this study. Therefore, the scale validated in this study should be considered as a contribution towards advancing the Uses and Grats 2.0 agenda put forward by Sundar and Limperos (2013), by situating the original items in a more strictly defined social media context.

The revised model has some deviations from the original framework. For instance, the results did not display several gratifications as distinct factors, suggesting the need to combine some factors. However, the combined factors did not include items from other affordances, supporting the conceptual accuracy of the original model. The question of operationalizing Coolness and Novelty emerges as a main topic for future investigation, as this study did not identify distinct factors for these two constructs. There are studies (e.g., Sundar et al., 2014) that show that Coolness is a complex construct that includes several dimensions. Possibly, a multidimensional Coolness measure can be integrated to the present model. Such an introduction, however, may be too abstract. On the other hand, there is a lack of conceptualization of the meaning of Novelty of social media. Accordingly, there is a need for a precise definition of Novelty and empirical work that can establish differences between Coolness and Novelty. 
Although Coolness and Novelty were excluded from the final scale, based on the argument that social media may not be novel anymore, these constructs can be applied in other settings. For instance, future work that examines uses and gratifications of new social media platforms as well as applications that integrate social media with technologies that users may perceive novel (e.g., virtual and augmented reality) can use Coolness and Novelty items as part of the scale.

A similar issue arises with regard to Browsing and Scaffolding, two constructs that this study did not differentiate between. However, as the focus of the scale here is to test an allinclusive model, conceptualizing Coolness and Novelty, and Browsing and Scaffolding/Navigation Aids, as single constructs do not jeopardize the value of the conceptualization. The fact that Ownness and Filtering were combined to form a single factor requires further study, as it is possible that these two constructs may form distinct factors if the measures are further refined. The scale can also benefit from further work that adds new constructs to the measure. For instance, device-based affordances, such as mobility, can be added to the conceptual framework, as mobile devices are frequently used to access social media. This can lead to unique gratifications, such as immediacy, and broaden the scope of the scale. Moreover, new constructs, such as social surveillance, can be added to the scale to capture the full potential of social media.

Despite the contribution this study makes by building on Sundar and Limperos's (2013) work, Cronbach's Alpha values lower than 0.7 for Bandwagon and Filtering indicate that these items should be tested further with different populations and larger samples to improve the internal consistency of the constructs. Moreover, the items removed in the data reduction process can be revised and tested with different samples. However, removal of these items does not affect the quality of the measure, as each latent construct was represented by at least three items. 
Items that measure Interaction, INTR1 and INTR3 in particular, should be subject to further revision and testing due to two reasons. First, Interaction is a main characteristic of social media and ignoring this construct may result in the scale not capturing the gratification of interaction. Further, two out of three items in the measure suggested indicated need for improvement (INTR1 low regression weight the in the CFA model, and INTR low factor loading). We have included Interaction items in the EFA and reported results of the first two CFAs with Interaction as it could provide a starting point for future researchers to refine the construct.

This study can form the foundation for a number of other studies. Future work should focus on differences in perception of social media uses and gratifications across different actors, such as political actors, and organizations. Similarly, effects of attributes, such as efficacy, tolerance, community engagement, openness, sociability, political orientation, and cynicism on social media uses and gratifications can be examined. Moreover, moderating effects of constructs such as internet skills, social media adoption, perceived internet controls, social surveillance, and privacy concerns can be tested to uncover nuances in relationships between actors, attributes, and social media uses and gratifications.

Future studies that examine social media uses and gratifications also need to consider differences between platforms. This study, as mentioned before, situates uses and gratifications in the context of social media that include several types of platforms. This is appropriate, as the MAIN framework considers new media as a single category that offers unique affordances that can lead to unique gratifications. Future studies can examine how platform type affects those gratifications. For instance, some social network sites may provide more community-building and interactivity-related gratifications than others. Moreover, the measure tested in this study can 
be further refined to develop uses and gratifications measures tailored to different types of platforms.

\section{References}

Alhabash, S., Chiang, Y., \& Huang, K. (2014). MAM \& U\&G in Taiwan: Differences in the uses and gratifications of Facebook as a function of motivational reactivity. Computers in Human Behavior, 35, 423-430. http://doi.org/10.1016/j.chb.2014.03.033

Ancu, M., \& Cozma, R. (2009). MySpace politics: Uses and Gratifications of befriending candidates. Journal of Broadcasting \& Electronic Media, 53, 567583. http://doi.org/10.1080/08838150903333064

Anderson, I. K. (2011). The Uses and Gratifications of online care pages: A study of CaringBridge. Health Communication, 26, 546-559. http://doi.org/10.1080/10410236.2011.558335

Apaolaza, V., He, J., \& Hartmann, P. (2014). The effect of gratifications derived from use of the social networking site Qzone on Chinese adolescents' positive mood. Computers in Human Behavior, 41, 203-211.

boyd, d. (2011). Social Network Sites as networked publics: Affordances, dynamics, and implications. In Z. Papacharissi (Ed.), A networked self: Identity, community, and culture on social network sites (pp. 39-58). New York and London: Routledge Taylor \& Francis Group. http://doi.org/10.4324/9780203876527

Chen, G. M. (2011). Tweet this: A uses and gratifications perspective on how active Twitter use gratifies a need to connect with others. Computers in Human Behavior, 27(2), 755762. http://doi.org/10.1016/j.chb.2010.10.023 
Chen, H. T., \& Kim, Y. (2013). Problematic use of social network sites: The interactive relationship between gratifications sought and privacy concerns. Cyberpsychology, Behavior and Social Networking, 16(11), 806-813. http://doi.org/10.1089/cyber.2011.0608

Hicks, A., Comp, S., Horovitz, J., Hovarter, M., Miki, M., \& Bevan, J. L. (2012). Why people use Yelp.com: An exploration of uses and gratifications. Computers in Human Behavior, 28(6), 2274-2279.http://doi.org/10.1016/j.chb.2012.06.034

Hu, L., \& Bentler, P. M. (1999). Cutoff criteria for fit indexes in covariance structure analysis: Conventional criteria versus new alternatives. Structural Equation Modeling: A Multidisciplinary Journal, 6(1), 1-55. http://doi.org/10.1080/10705519909540118

Huang, L.Y., Hsieh, Y.-J., \& Wu, Y.-C. J. (2014). Gratifications and social network service usage: The mediating role of online experience. Information \& Management, 51, 774-782.

Kaye, B. K. (2010). Going to the blogs: Toward the development of a Uses and Gratifications measurement scale for blogs. Atlantic Journal of Communication, 18, 194-210. http://doi.org/10.1080/15456870.2010.505904

Kim, J. W. (2014). Scan and click: The uses and gratifications of social recommendation systems. Computers in Human Behavior, 33, 184-191. http://doi.org/10.1016/j.chb.2014.01.028

Krause, A. E., North, A. C., \& Heritage, B. (2014). The uses and gratifications of using Facebook music listening applications. Computers in Human Behavior, 39, 7177. http://doi.org/10.1016/j.chb.2014.07.001 
Leung, L. (2013). Generational differences in content generation in social media: The roles of the gratifications sought and of narcissism. Computers in Human Behavior, 29(3), 997-1006. http://doi.org/10.1016/j.chb.2012.12.028

Luo, M. M., \& Remus, W. (2014). Uses and gratifications and acceptance of Web-based information services: An Integrated Model. Computers in Human Behavior, 38, 281295.

Majchrzak, A., Faraj, S., Kane, G. C., \& Azad, B. (2013). The contradictory influence of social media affordances on online communal knowledge sharing. Journal of Computer-Mediated Communication, 19(1), 38-55. http://doi.org/10.1111/jcc4.12030

Pai, P., \& Arnott, D. C. (2013). User adoption of social networking sites: Eliciting uses and gratifications through a means-end approach. Computers in Human Behavior, 29(3), 1039-1053. http://doi.org/10.1016/j.chb.2012.06.025

Park, N., Kee, K. F., \& Valenzuela, S. (2009). Being immersed in social networking environment: Facebook groups, Uses and Gratifications, and social outcomes. Cyberpsychology \& Behavior, 12(6), 729-733.

Quan-Haase, A., \& Young, A. L. (2010). Uses and Gratifications of social media: A comparison of Facebook and instant messaging. Bulletin of Science, Technology \& Society, 30(5), 350-361. http://doi.org/10.1177/0270467610380009

Roy, S. K. (2009). Internet uses and gratifications: A survey in the Indian context. Computers in Human Behavior, 25(4), 878-886. http://doi.org/10.1016/j.chb.2009.03.002

Ruggiero, T. E. (2000). Uses and Gratifications Theory in the 21st century. Mass Communication and Society, 3(1), 3-37. http://doi.org/10.1207/S15327825MCS0301_02 
Smock, A. D., Ellison, N. B., Lampe, C., \& Wohn, D. Y. (2011). Facebook as a toolkit: A uses and gratification approach to unbundling feature use. Computers in Human Behavior, 27(6), 2322-2329. http://doi.org/10.1016/j.chb.2011.07.011

Sundar, S. S. (2008). The MAIN model: A heuristic approach to understanding technology effects on credibility. In M. J. Metzger \& A. J. Flanagin (Eds.), Digital media, youth, and credibility (pp. 73-100). Cambridge, MA: The MIT Press. http://doi.org/10.1162/dmal.9780262562324.073

Sundar, S. S., \& Limperos, A. M. (2013). Uses and Grats 2.0: New gratifications for new media. Journal of Broadcasting \& Electronic Media, 57(4), 504-525. http://doi.org/10.1080/08838151.2013.845827

Sundar, S. S., Tamul, D. J., \& Wu, M. (2014). Capturing “cool”: Measures for assessing coolness of technological products. International Journal of Human-Computer Studies, 72, 169-180. http://doi.org/10.1016/j.ijhcs.2013.09.008

Wang, Z., Tchernev, J. M., \& Solloway, T. (2012). A dynamic longitudinal examination of social media use, needs, and gratifications among college students. Computers in Human Behavior, 28(5), 1829-1839.

Wu, J.-H., Wang, S.-C., \& Tsai, H.-H. (2010). Falling in love with online games: The uses and gratifications perspective. Computers in Human Behavior, 26(6), 1862-1871. http://doi.org/10.1016/j.chb.2010.07.033

Zhang, L., \& Zhang, W. (2013). Real-time Internet news browsing: Information vs. experience- related gratifications and behaviors. Computers in Human Behavior, 29(6), 2712-2721. http://doi.org/10.1016/j.chb.2013.07.009 

Table 1. 16 affordances related to four broad classes (Sundar \& Limperos, 2013)

\begin{tabular}{llll}
\hline Modality & Agency & Interactivity & Navigablity \\
\hline Realism & Agency-enhancement & Interaction & Browsing/Variety-seeking \\
Coolness & Community building & Activity & Scaffolds/Navigation aids \\
Novelty & Bandwagon & Responsiveness & Play/Fun \\
Being there & Filtering/Tailoring & Dynamic control & \\
& Ownness & & \\
& & & \\
\hline
\end{tabular}


Table 2. Factor loadings

\begin{tabular}{|c|c|c|c|c|c|}
\hline Construct & Item & $\begin{array}{l}\text { Item } \\
\text { Mean }\end{array}$ & $\begin{array}{l}\text { Item } \\
\text { SD }\end{array}$ & $\begin{array}{l}\text { Factor } \\
\text { Loading }\end{array}$ & $\begin{array}{l}\text { Construct } \\
\text { Mean, SD, } \\
\text { and } \alpha\end{array}$ \\
\hline \multicolumn{6}{|l|}{ EFA 1} \\
\hline \multirow[t]{3}{*}{ Realism } & $\begin{array}{l}\text { REAL2- Communicating using social media is not that } \\
\text { different from face-to-face communication. }\end{array}$ & 1.924 & .886 & .580 & \multirow{3}{*}{$\begin{array}{l}\text { Mean: } 2.03 \\
\text { SD: .738 } \\
\alpha: .705\end{array}$} \\
\hline & $\begin{array}{l}\text { REAL3- The experience in social media is very much like } \\
\text { real life. }\end{array}$ & 1.939 & .936 & .857 & \\
\hline & $\begin{array}{l}\text { REAL4- I can experience the real world through social } \\
\text { media. }\end{array}$ & 2.215 & 1.020 & .556 & \\
\hline \multirow[t]{5}{*}{ Coolness } & $\begin{array}{l}\text { COOL1- Social media platforms are unique compared to } \\
\text { other media. }\end{array}$ & 3.697 & .826 & .816 & \multirow{5}{*}{$\begin{array}{l}\text { Mean:3.75 } \\
\text { SD: .589 } \\
\alpha: .762\end{array}$} \\
\hline & $\begin{array}{l}\text { COOL2- Social media platforms are distinctive compared } \\
\text { to other media. }\end{array}$ & 3.711 & 0.726 & .740 & \\
\hline & $\begin{array}{l}\text { NOVL1- Social media platforms are new compared to } \\
\text { other media. }\end{array}$ & 3.708 & .821 & .459 & \\
\hline & NOVL2- Social media platforms have innovative features. & 3.841 & .674 & .508 & \\
\hline & $\begin{array}{l}\text { NOVL3- Social media interfaces are different than } \\
\text { traditional websites. }\end{array}$ & 3.844 & .684 & .506 & \\
\hline \multirow{3}{*}{$\begin{array}{l}\text { Being } \\
\text { There }\end{array}$} & BEIN1- Social media help me immerse myself in places & 3.443 & 1.009 & .722 & \multirow{3}{*}{$\begin{array}{l}\text { Mean: } 3.25 \\
\text { SD: } .886 \\
\alpha: .802\end{array}$} \\
\hline & that I cannot physically experience. & & & & \\
\hline & $\begin{array}{l}\text { BEIN2- Social media create the experience of being } \\
\text { present in distant environments. }\end{array}$ & 3.314 & 1.032 & .811 & \\
\hline
\end{tabular}


BEIN3- Social media provide quality information that 2.991 1.016 .723 makes me feel like I am able to experience things as they

EFA 2 are without actually being there.

Agency BEIN5-Social media help me to have real interactions with people although I am not in physical proximity

AGNC1- Social media allow me to freely express my 3.566

$3.373 \quad 1.049$

.423

opinions.

AGNC2- Social media allow me to freely assert my

$\quad 918$

Mean: 3.58

identity.

AGNC5- Social media allow me to have my say.

$3.483 \quad .897 \quad .774$

SD: .759

AGNC5-Social media allow me to have my say.

3.696

.812

Community CMNB2-Social media help me to be part of a community

3.710

.896

$\alpha: .832$

Building

that I would not otherwise have been part of.

CMNB3- Social media allow me to build a network that

could bring me social support.

CMNB4- Social media allow me to actively con
communities that make an impact on society.

SD: .712

$\alpha: .787$

Bandwagon BAND2- Social media comfort me by letting me know the 
BAND4- Reading others comments on social media before

3.636

.893

.684

$\alpha: .684$

I make comments helps me to avoid potential conflicts.

$\begin{array}{lllll}\text { BAND5- I try to adjust my reactions to social media posts } & 3.056 & 1.037 & .723\end{array}$

based on comments made by others.

Filtering

FILT3- Social media allow me to sort through information before I share it with others.

FILT4- Social media allow me to limit the visibility of

.768

.560

Mean: 3.83

information I post to a small group.

$\begin{array}{lll}3.741 & .889 & .709\end{array}$

SD: .545

OWNN3- My friends have their own ways of using social

4.064

.637

$\alpha$ : .640

media.

EFA 3

Interaction INTR1- Social media rely on user interaction

.560

Mean: 3.70

INTR2- When I use social media, I frequently interact with the platform

.896

.536

SD: .603

INTR3- On social media, I can specify my needs and preferences on an ongoing basis

$\alpha: .570$

Activity ACTV1- I can perform a number of tasks on social media.

3.680

.765

.526

Mean:3.08

ACTV2- I feel active when I use social media.

2.910

1.069

.679 
ACTV3- My Interaction on social media is not passive.

3.019

.912

.606

$\alpha: .775$

ACTV4- I get to do a lot of things on social media.

$3.309 \quad .960$

.655

Responsive RESP2-Social media are responsive to my commands.

ness

RESP3- Social media respond well to my requests.

$3.202 \quad .856 \quad .706$

Mean: 3.12

RESP3-Social media respond well to my requests.

DYNM1- Social media allow me to be in charge.

$3.201 \quad .822 \quad .783$

SD: .744

$\alpha: .835$

DYNM2- Social media give me more control over

$2.971 \quad .947 \quad .595$

information I post.

$3.550 \quad .817 \quad .568$

DYNM3- I am able to control my interaction with the

$3.550 \quad .771$

.605

interfaces of the social media platforms I use.

EFA 4

$\begin{array}{llllll}\text { Browsing } & \begin{array}{l}\text { BROW1- Social media allow me to obtain a wide variety } \\ \text { of information. }\end{array} & 3.776 & .844 & .782 & \begin{array}{l}\text { Mean: } 3.80 \\ \text { SD: } .629 \\ \alpha: .880\end{array} \\ \begin{array}{l}\text { BROW2- Social media can link me to sites that have } \\ \text { different types of information. }\end{array} & 3.933 & .702 & .758 & \\ & \begin{array}{l}\text { BROW3- Social media allow me to surf for things that I am } \\ \text { interested in. }\end{array} \\ & 3.645 & .766 & .774 \\ \text { BROW4- Social media allow me to browse freely. } & 3.736 & .828 & .590\end{array}$


SCAF2- Social media allow me to link to other pieces of

information.

SCAF3- Social media offer a number of visual aids for

more effective use.

Play PLAY3- I enjoy escaping into a different world through

3.347

1.006

.525

Mean: 3.50 social media.

PLAY4- Social media provide more entertaining

888

SD: .778

information than other media.

$\alpha: .805$

PLAY5- Social media offer more entertaining features than 3.609 
Table 3. Correlation matrix

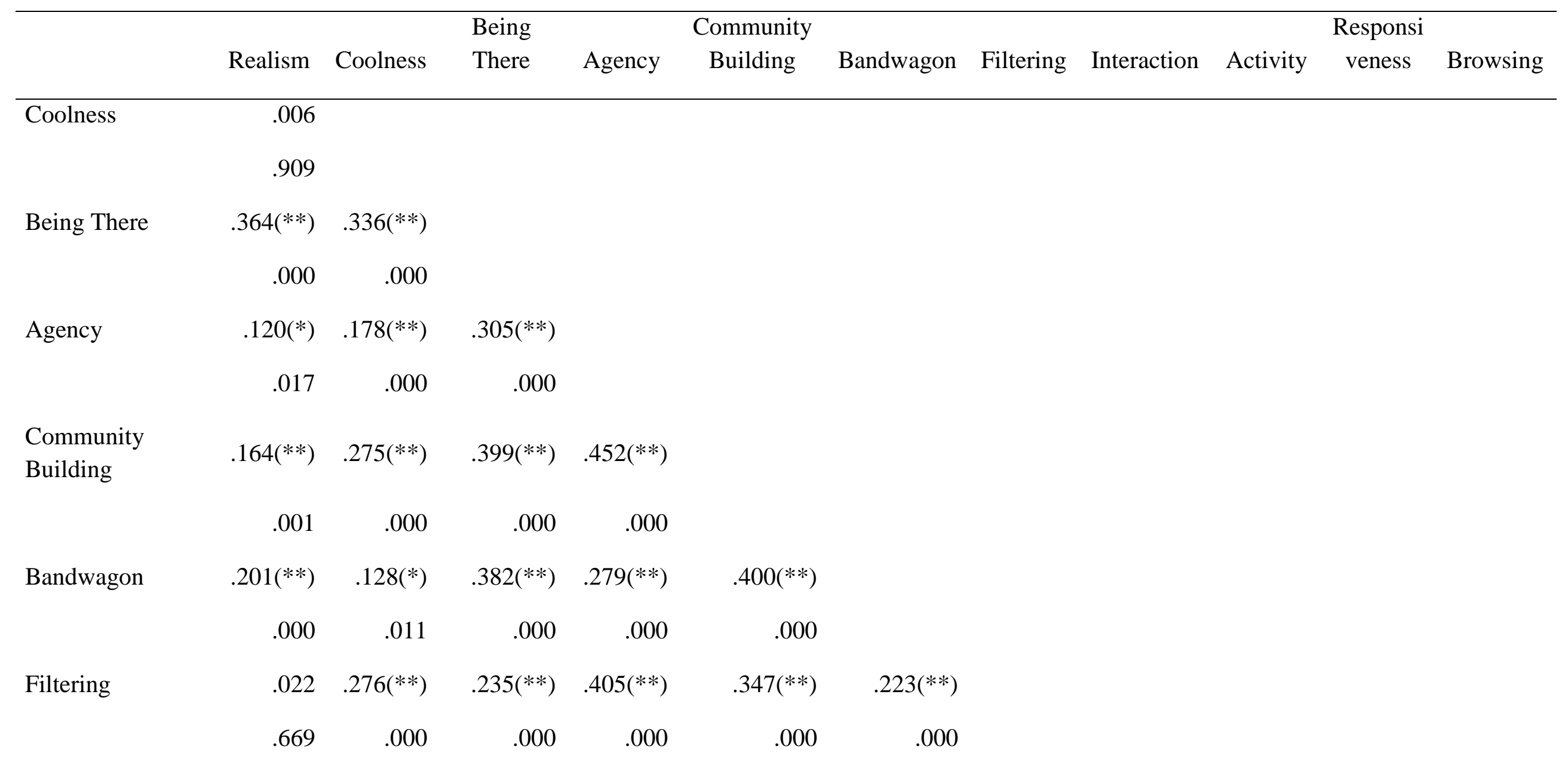




\begin{tabular}{|c|c|c|c|c|c|c|c|c|c|c|c|}
\hline \multirow[t]{2}{*}{ Interaction } & .066 & $.302(* *)$ & $.249(* *)$ & $.332(* *)$ & $.371(* *)$ & $.252(* *)$ & $.411(* *)$ & & & & \\
\hline & .192 & .000 & .000 & .000 & .000 & .000 & .000 & & & & \\
\hline \multirow[t]{2}{*}{ Activity } & $.328(* *)$ & $.207(* *)$ & $.416(* *)$ & $.373(* *)$ & $.403(* *)$ & $.446(* *)$ & $.227(* *)$ & $.391(* *)$ & & & \\
\hline & .000 & .000 & .000 & .000 & .000 & .000 & .000 & .000 & & & \\
\hline \multirow[t]{2}{*}{ Responsiveness } & $.185(* *)$ & $.202(* *)$ & $.407(* *)$ & $.365(* *)$ & $.370(* *)$ & $.370(* *)$ & $.316(* *)$ & $.381(* *)$ & $.568(* *)$ & & \\
\hline & .000 & .000 & .000 & .000 & .000 & .000 & .000 & .000 & .000 & & \\
\hline \multirow[t]{2}{*}{ Browsing } & .066 & $.369(* *)$ & $.346(* *)$ & $.284(* *)$ & $.407(* *)$ & $.364(* *)$ & $.421(* *)$ & $.498(* *)$ & $.360(* *)$ & $.444(* *)$ & \\
\hline & .193 & .000 & .000 & .000 & .000 & .000 & .000 & .000 & .000 & .000 & \\
\hline \multirow[t]{2}{*}{ Play } & $.207(* *)$ & $.233(* *)$ & $.378(* *)$ & $.378(* *)$ & $.344(* *)$ & $.469(* *)$ & $.246(* *)$ & $.394(* *)$ & $.455(* *)$ & $.372(* *)$ & $.442(* *)$ \\
\hline & .000 & .000 & .000 & .000 & .000 & .000 & .000 & .000 & .000 & .000 & .000 \\
\hline
\end{tabular}

** Correlation is significant at the 0.01 level (2-tailed).

* Correlation is significant at the 0.05 level (2-tailed). 
Table 4. Latent factor structure

\begin{tabular}{llll}
\hline Modality & Agency & Interactivity & Navigability \\
\hline Realism & Agency-enhancement & Interaction & Browsing \\
Being There & Community Building & Activity & Play \\
& Bandwagon & Responsiveness & \\
& Filtering & & \\
& & \\
\end{tabular}


Figure 1: Social media Uses and Gratifications scale as a second-order factor structure

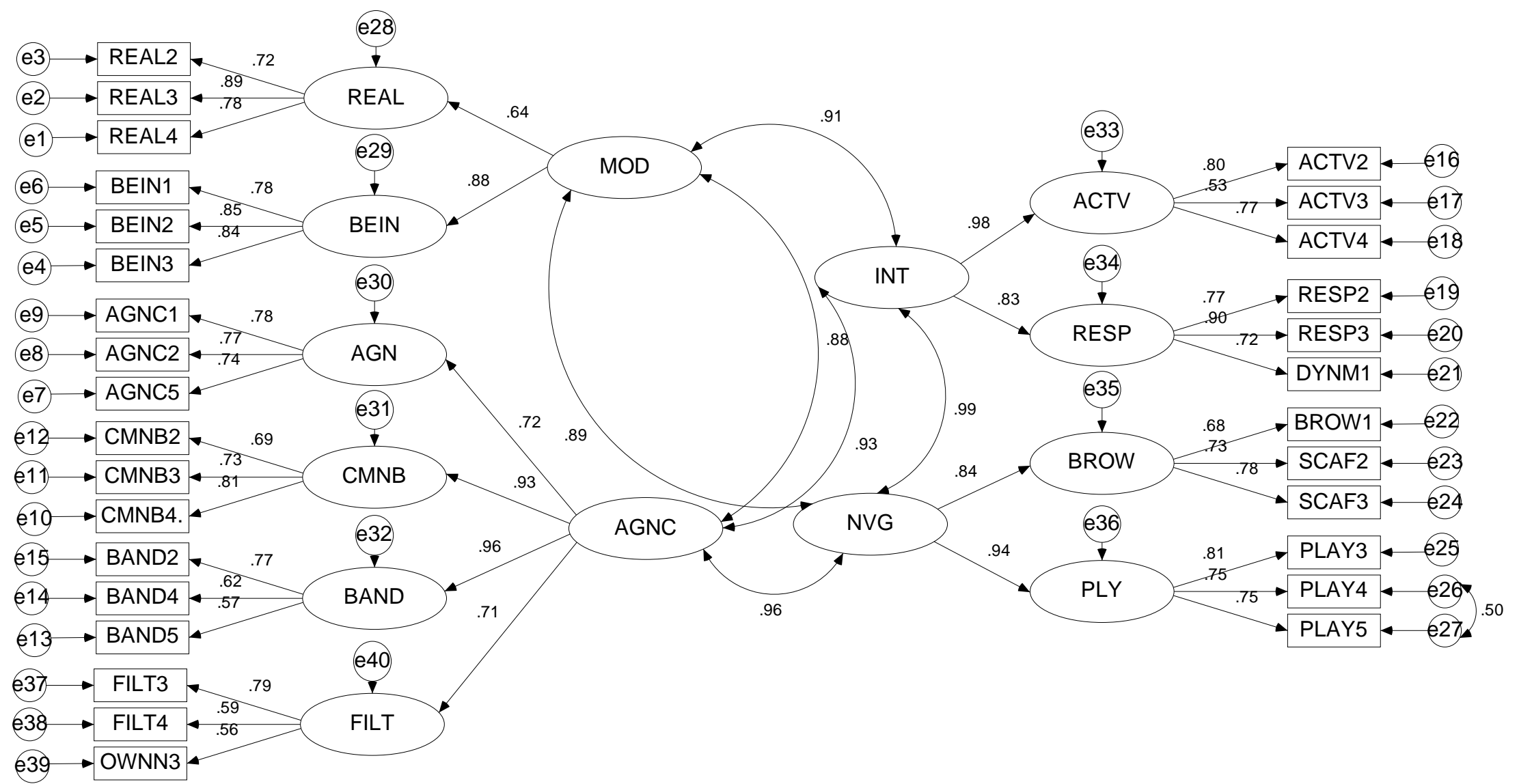

Model fit: Chi-square $=740.944, d f: 388, p: 0.000$, RMR: 0.048, GFI: 0.852, AGFI: 0.822, IFI: 0.931, TLI: 0.922, CFI: 0.931, RMSEA: 0.054 